\title{
Scaling Symmetries of Scatterers of Classical Zero-Point Radiation
}

\author{
Timothy H. Boyer \\ Department of Physics, City College of the City \\ University of New York, New York, New York 10031
}

\begin{abstract}
Classical radiation equilibrium (the blackbody problem) is investigated by the use of an analogy. Scaling symmetries are noted for systems of classical charged particles moving in circular orbits in central potentials $V(r)=-k / r^{n}$ when the particles are held in uniform circular motion against radiative collapse by a circularly polarized incident plane wave. Only in the case of a Coulomb potential $n=1$ with fixed charge $e$ is there a unique scale-invariant spectrum of radiation versus frequency (analogous to zero-point radiation) obtained from the stable scattering arrangement. These results suggest that non-electromagnetic potentials are not appropriate for discussions of classical radiation equilibrium.
\end{abstract}




\section{INTRODUCTION}

The blackbody problem within classical physics has never been solved. Solution requires accurate calculations determining the spectra of random classical radiation which are in steady-state equilibrium with various classical mechanical scattering systems. Calculations for a few scattering potentials have been performed. Scattering calculations involving the dipole approximation for particles of very small charge in a harmonic potential[1] or in some nonlinear potentials have been carried out.[2][3] Although the harmonic potential gives no condition on the radiation spectrum other than spatial isotropy, the other potentials suggest that the Rayleigh-Jeans spectrum is the only radiation spectrum giving equilibrium between radiation and matter in classical physics. Also, a class of potentials has been treated when the scattering particle's linear momentum takes the relativistic form, [4] and again the conclusion was that equilibrium required the Rayleigh-Jeans spectrum.

It has been suggested for more than thirty years [3] that understanding of classical radiation equilibrium may require the use of relativistic scattering systems, specifically particles in Coulomb potentials. More recently it has been noted that the unique value $e$ of electronic charge may also play a crucial role in classical radiation equilibrium. [5] In the last two decades, these suggestions have been been greeted with overwhelming rejection by the

referees at the leading physics journals. One referee declared that these suggestions are merely desperate searches for "loopholes" to avoid the obvious conclusion that classical physics is associated with the Rayleigh-Jeans spectrum. Other referees have suggested that such ideas are so far from currently accepted physics that they were unpublishable unless a complete solution of the classical blackbody problem (and indeed of all atomic physics) was presented.

Nevertheless the fact remains that there has never been a classical scattering calculation involving the Coulomb potential, which is the only classical potential which has been extended to a fully relativistic system. Now the Coulomb potential is special not only because of its connections to relativistic physics but also because of its scaling symmetry properties. Since most physicists seem unable to take seriously the need for relativistic scattering systems for blackbody radiation equilibrium, perhaps scaling symmetry (which is easy to analyze) may afford an easier glimpse into the problems of classical radiation equilibrium. A scaling analysis of scattering is what we carry out in this article. We 
carry out precise calculations for a model scattering situation and note the scale invariance and universal character of the radiation spectrum associated with scattering involving the Coulomb potential and only the Coulomb potential. We believe the results indicate the serious nature of the remaining "loophole" in the classical blackbody problem.

\section{CLASSICAL ELECTROMAGNETIC ZERO-POINT RADIATION}

In this article we carry out a model scattering calculation which represents a crude analogue to treating zero-temperature thermal radiation, classical electromagnetic zero-point radiation. Now apparently many physicists are still unaware of the concept of classical zero-point radiation, and indeed some referees at the leading physics journals regularly reject the possibility of classical electromagnetic zero-point radiation. However, classical zero-point radiation is an intrinsic part of classical electromagnetism which enters the theory as the homogeneous boundary condition on Maxwell's equations. 11] It is required in order to give a classical electromagnetic description of the experimentally observed van der Waals forces (Casimir forces) between macroscopic objects at zero temperature.[6] Furthermore, classical electromagnetic zero-point radiation in connection with electric dipole harmonic oscillator systems provides a classical description of a number of phenomena which are usually regarded as having only a quantum description, such as van der Waals forces, [1] diamagnetism, [7] and thermal effects of acceleration through the vacuum. [8] A reader of this manuscript who can not conceive of classical electromagnetic zero-point radiation may find it hard to follow the logic of our model calculation. The decision to restrict our analogy to zero-temperature was made so as to simplify the analysis as much as possible and thereby hopefully make it transparent to readers.

Classical zero-point radiation is the random classical radiation which is present at the absolute zero of temperature. In order to fit the experimentally observed Casimir forces (as calculated numerous times [9] under various conditions within classical electrodynamics), it must take the form

$$
E_{\omega}=b_{z p} \omega
$$

where $E_{\omega}$ is the average energy per normal mode of angular frequency $\omega$, and $b_{z p}$ is a constant. For numerical agreement with the observed Casimir forces, the constant $b_{z p}$ must 
take the value

$$
b_{z p}=5.27 \times 10^{-28} \mathrm{erg}-\mathrm{sec}
$$

which is a familiar number.[10] The spectrum of zero-point radiation is (up to a multiplicative constant) the unique spectrum of random classical radiation which is Lorentz invariant[11] and the unique spectrum which is scale invariant[12] under the $\sigma_{l t E^{-1-s c a l i n g}}$ allowed by electromagnetism. [13] Since each normal mode of the electromagnetic field at (angular) frequency $\omega$ takes the form of a harmonic oscillator 14] at frequency $\omega$ and can be describable in terms of action-angle variables, it follows from Eq. (1) and $E=J_{\omega} \omega$ that the average value of the action variable $J_{\omega}$ (associated with each normal mode of frequency $\omega)$ takes the same constant value $b_{z p}$ independent of $\omega$.

Since classical electromagnetic radiation in an enclosure can not bring itself to equilibrium, the interaction of radiation with scattering systems represents the crucial element in determining the equilibrium spectrum. Thermal radiation equilibrium involves steady-state behavior for both the scatterer and the radiation. The motion of the charged particles in matter and the random radiation must fit together so that, on average, there is no change in the particle energy and no change in the spectrum of the random radiation. Presumably there is some aspect encoded within the scatterers which produces the universal character found for thermal radiation. In this article we investigate the scattering of radiation by charged particles in a crude model and find that the Coulomb potential possesses scaling aspects which allow an associated universal radiation spectrum.

\section{THE MODEL CALCULATION}

In our simplified model, we consider a single charged particle of mass $m$ and charge $e$ undergoing uniform circular motion with speed $v=\omega r$ in a central potential $V(r)=-k / r^{n}$, the motion satisfying Newton's second law

$$
m \gamma \frac{v^{2}}{r}=n \frac{k}{r^{n+1}}
$$

where $\gamma=\left(1-v^{2} / c^{2}\right)^{-1 / 2}$. The orbital angular momentum of the particle is given by

$$
J=r m \gamma v
$$


Now if the particle undergoing uniform circular motion has charge $e$, then it will radiate energy into the electromagnetic field at the rate 15$]$

$$
P=\frac{2}{3} \frac{e^{2}}{c^{3}} \omega^{4} \gamma^{4} r^{2}
$$

In order to balance the energy loss to emitted electromagnetic radiation, we imagine a circularly polarized plane wave of angular frequency $\omega$ propagating along the axis perpendicular to the orbital motion. (We may picture the particle's motion as constrained by two frictionless plane sheets so that it remains in a fixed orbital plane despite the magnetic force due to the plane wave, or else we can consider two counter-propagating plane waves so as to eliminate the magnetic field in the orbital plane.) The amplitude $\mathcal{E}_{0}$ of the electric field of the plane wave is taken as the smallest possible value such that the incident wave will provide the energy loss given in Eq. (5). Thus we require

$$
P=\frac{2}{3} \frac{e^{2}}{c^{3}} \omega^{4} \gamma^{4} r^{2}=e \mathcal{E}_{0} v
$$

or, since $r=\omega / v$,

$$
\mathcal{E}_{0}=\frac{2}{3} \frac{e}{c^{3}} \omega^{2} v \gamma^{4}
$$

with an associated radiation energy density $u$ for the incident plane wave

$$
u=\frac{1}{8 \pi}\left(\mathcal{E}_{0}^{2}+\mathcal{B}_{0}^{2}\right)=\frac{1}{9 \pi} \frac{e^{2}}{c^{6}} \omega^{4} v^{2} \gamma^{8}
$$

In this fashion we make an association between a particle motion and and an electromagnetic field corresponding to a steady-state coherent (in contrast to random) radiation scattering situation. This association can be regarded as an analogue of the association between the average particle motion and the electromagnetic spectrum which holds in classical thermal radiation equilibrium. Since both scattering situations involve the known classical electromagnetic interaction between charged particles and electromagnetic waves, we expect that exploring one situation may give us insights into the other.

\section{IV. $\sigma_{l t E^{-1}}$-SCALING SYMMETRY FOR CLASSICAL ELECTROMAGNETISM}

We say that a system is $\sigma$-scale invariant or has $\sigma$-scaling symmetry if the system is mapped onto itself under a dilatation which multiplies appropriate quantities in the system by a factor of $\sigma$. Although the set of all classical mechanical systems allows independent 
scalings of length $\sigma_{l}$, time $\sigma_{t}$, and energy $\sigma_{E}$, classical electromagnetism allows only one

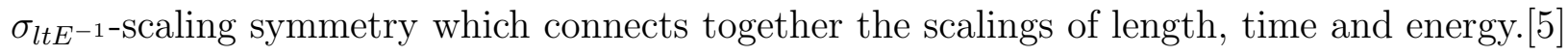
The scaling coupling of length and time is required by the appearance of a fundamental velocity $c$ for electromagnetic radiation, and the coupling of energy and length is required by the appearance of a smallest elementary charge $e$ in nature. Under $\sigma_{l t E^{-1} \text {-scaling, }}$ lengths are scaled as $l \rightarrow l^{\prime}=\sigma_{l t E^{-1}} l$, times are scaled as $t \rightarrow t^{\prime}=\sigma_{l t E^{-1}} t$, and energies

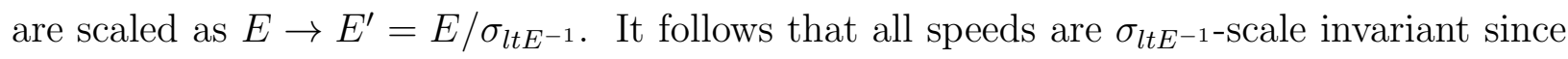
$v=l / t \rightarrow v^{\prime}=\left(\sigma_{l t E^{-1}} l\right) /\left(\sigma_{l t E^{-1}} t\right)=l / t=v$. Electric charge $q$ is $\sigma_{l t E^{-1} \text {-scale invariant since }}$ the potential energy $E$ between two point charges $q$ a distance $r$ apart behaves as $q^{2}=E r \rightarrow$ $q^{\prime 2}=E^{\prime} r^{\prime}=\left(E / \sigma_{l t E^{-1}}\right)\left(\sigma_{l t E^{-1}} r\right)=q^{2}$. Orbital angular momentum $J$ is $\sigma_{l t E^{-1} \text {-scale invariant }}$ since $J=r m \gamma v \rightarrow J^{\prime}=r^{\prime} m^{\prime} \gamma^{\prime} v^{\prime}=\left(\sigma_{l t E^{-1}} r\right)\left(m / \sigma_{l t E^{-1}}\right) \gamma v=r m \gamma v=J$. Electromagnetic

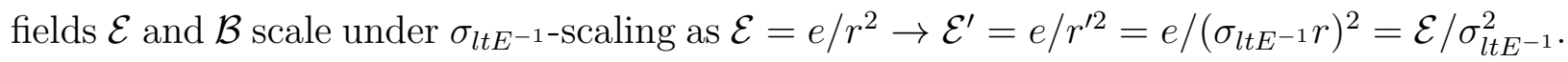
Mass $m$ is related to energy $m c^{2}$ and so scales as an energy $m \rightarrow m^{\prime}=m / \sigma_{l t E^{-1}}$.

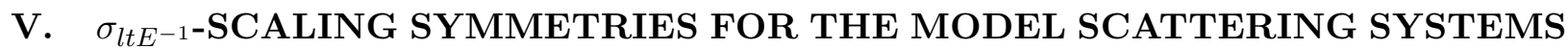

We are now in a position to note the scaling symmetries for the model scattering systems

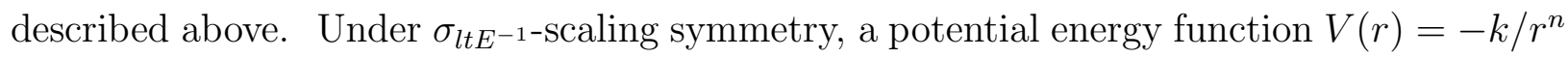
is mapped to a new potential energy function $V(r) \rightarrow V^{\prime}\left(r^{\prime}\right)=V(r) / \sigma_{l t E^{-1}}=-k^{\prime} /\left(r^{\prime}\right)^{n}=$ $-k^{\prime} /\left(\sigma_{l t E^{-1}} r\right)^{n}=-\sigma_{l t E^{-1}}^{-n} k^{\prime} / r^{n}=-\left(\sigma_{l t E^{-1}}^{-n} k^{\prime} / k\right) k / r^{n}$, or $V(r)=-\left(\sigma_{l t E^{-1}}^{1-n} k^{\prime} / k\right) k / r^{n}$. Form invariance requires that

$$
k^{\prime}=\sigma_{l t E^{-1}}^{n-1} k
$$

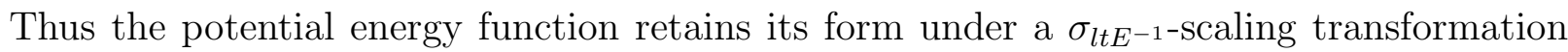
if and only if the constant $k$ appearing in the potential energy is also transformed to $k^{\prime}=$ $\sigma_{l t E^{-1}}^{n-1} k$. Thus there is exactly one potential energy function which is $\sigma_{l t E^{-1} \text {-scale invariant }}$ $k^{\prime}=k$, and that is the potential energy function where $n=1$, namely the Coulomb potential $V(r)=-k / r$. If we write the potential function in terms of elementary charges of magnitude $e$, this is $V(r)=-e^{2} / r$.

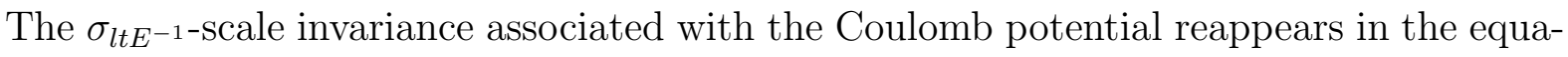
tion for the orbital speed of the particle. Thus combining equations (3) and (4) for a general potential $V(r)=-k / r^{n}$, we can eliminate the orbital radius $r$ so as to obtain an equation connecting the orbital speed $v$ and the orbital angular momentum $J$ in terms of the particle 
mass $m$ and the potential parameter $k$

$$
v^{2-n} \gamma^{1-n}=\frac{n k}{J^{n}} m^{n-1}
$$

The Coulomb potential with $n=1$ is very special because in this case (and only in this case) the particle mass $m$ disappears from Eq. (10) for the orbital speed giving

$$
v=\frac{k}{J}=\frac{e^{2}}{J}
$$

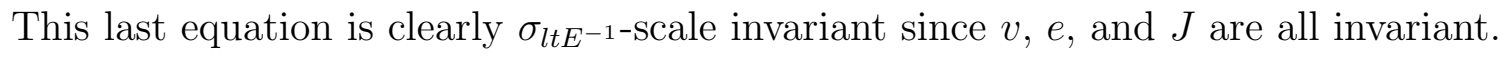

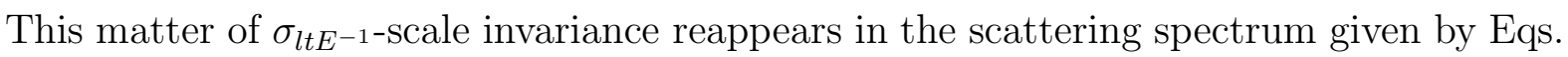
(7) and (8) and gives a universal character to the spectrum. The electric field $\mathcal{E}_{0}$ appearing in Eq. (7) can be regarded as a function of the angular frequency $\omega$ of the wave (which exactly matches the angular frequency of the orbital motion of the mass $m$ in the potential $V(r)$ ) and of the orbital particle speed $v$. For the Coulomb potential (and only for the Coulomb potential), the orbital speed $v$ as given in Eq. (11) is independent of the particle mass $m$. Thus the equations Eq. (7) and (8) for the electric field and the electromagnetic energy density become

$$
\mathcal{E}_{0}=\frac{2}{3} \frac{e}{c^{3}} \omega^{2}\left(\frac{e^{2}}{J}\right)\left[1-\left(\frac{e^{2}}{J c}\right)^{2}\right]^{-2}
$$

and

$$
u=\frac{1}{8 \pi}\left(\mathcal{E}_{0}^{2}+\mathcal{B}_{0}^{2}\right)=\frac{1}{9 \pi} \frac{e^{2}}{c^{6}} \omega^{4}\left(\frac{e^{2}}{J}\right)^{2}\left[1-\left(\frac{e^{2}}{J c}\right)^{2}\right]^{-4}
$$

We notice that the radiation spectrum given by Eqs. (7) and (8) makes no reference whatsoever to the orbital motion of any charged particle $m$ other than through the angular momentum $J$. The spectrum associates an electric field $\mathcal{E}_{0}$ and corresponding electromagnetic energy density $u$ with a given frequency $\omega$ and with fundamental constants $e$ and $c$ provided that the value of any orbital angular momentum $J$ is chosen as a constant, perhaps chosen as the same constant as appeared above in Eq. (2). Thus we have arrived at a universal spectrum of just the sort which we would want to associate with thermal radiation at zero temperature, classical electromagnetic zero-point radiation. We also emphasize that the constant $k=e^{2}$ can not be allowed to change continuously or the universal character of the spectrum will be lost.

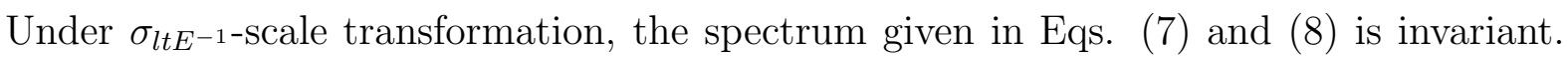
The electric field transforms as $\mathcal{E}_{0} \rightarrow \mathcal{E}_{0}^{\prime}=\mathcal{E}_{0} / \sigma_{l t E^{-1}}^{2}$ while the energy per unit volume 
transforms as $u \rightarrow u^{\prime}=u / \sigma_{l t E^{-1}}^{4}$; these transformation forms are matched by the powers of $\omega$ appearing on the right-hand sides of the equations while all the remaining parameters are $\sigma_{l t E^{-1} \text {-scale invariant. }}$

Perhaps it is helpful to characterize the uniqueness of the scattering spectrum (7) in the

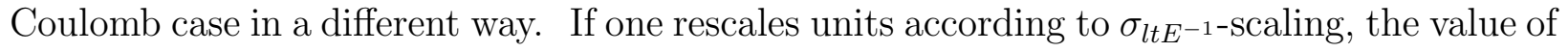
$k$ appearing in the potential energy function $V(r)=-k / r^{n}$ changes with the units for every case except $n=1$. Thus it is natural to regard $k$ as a parameter available for an adiabatic change in mechanics, except for the Coulomb potential function where the parameter $e^{2}=k$


in orbit in the potential $V(r)$ is an action variable and so is an adiabatic invariant which does not change with an adiabatic change of the parameter $k$. Thus for a general value of $n$, the different values of $k$ for fixed mass $m$ will involve different frequencies $\omega$ and different scattering electric fields $\mathcal{E}_{0}$. This generates a spectrum connecting electric field strength $\mathcal{E}_{0}$ and frequency $\omega$ for each fixed value of mass $m$. However, in general, different values of mass $m$ will generate different spectral connections between $\mathcal{E}_{0}$ and $\omega$. Now we remember that thermal radiation equilibrium involves a unique equilibrium spectrum which is independent of the mass $m$ of the charged particle which is scattering the radiation. In our crude analogue to thermal radiation scattering, we have found that the Coulomb potential energy function with unique charge $e$ is associated with a unique spectrum connecting $\mathcal{E}_{0}$ and $\omega$ independent of the mass $m$ of the orbiting particle. Moreover, the Coulomb scattering potential is the only potential energy function of the form $V(r)=-k / r^{n}$ which gives such a unique spectrum.

\section{SCALING SYMMETRIES FOR SCATTERERS OF CLASSICAL ZERO- POINT RADIATION}

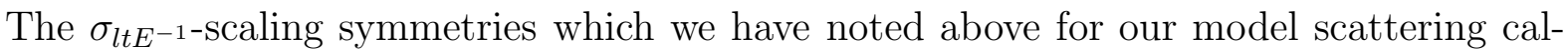
culation reappear in the scattering of random radiation at zero-temperature corresponding to classical electromagnetic zero-point radiation. The zero-point radiation spectrum is 


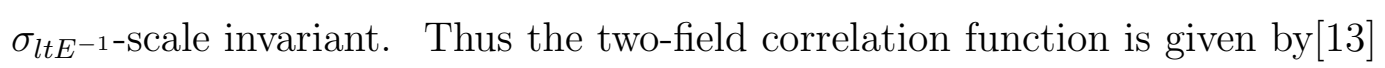

$$
\begin{aligned}
& \left\langle F_{z p}^{\mu \nu}(x) F_{z p}^{\sigma \rho}(y)\right\rangle \\
& =\left(g^{\mu \sigma} \partial_{x}^{\nu} \partial_{y}^{\rho}-g^{\mu \rho} \partial_{x}^{\nu} \partial_{y}^{\sigma}-g^{\nu \sigma} \partial_{x}^{\mu} \partial_{y}^{\rho}+g^{\sigma \rho} \partial_{x}^{\mu} \partial_{y}^{\nu}\right) \frac{2 b_{z p} c}{\pi(x-y)^{2}}
\end{aligned}
$$

where $F_{z p}^{\mu \nu}(x)$ is the electromagnetic field tensor, $g^{\mu \nu}$ is the metric for Minkowski spacetime, $x$ and $y$ are spacetime displacements, and $b_{z p}$ is the constant appearing in Eq. (2) which

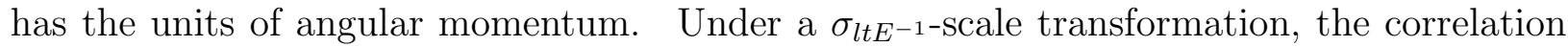
function is invariant [13] since the the four inverse powers of $\sigma_{l t E^{-1}}$ associated with the two factors of electromagnetic field on the left-hand side are matched by the four inverse powers of space or time on the right-hand side.

The scattering equations for a particle of charge $e$ in a Coulomb potential function $V(r)=$ $-e^{2} / r$ can be given in manifestly Lorentz-covariant form. The particle motion follows the relativistic form of Newton's second law including radiation reaction (which is usually known as the Lorentz-Dirac equation) [16]

$$
m \frac{d^{2} x^{\mu}}{d \tau^{2}}=e F_{\text {Coul }}^{\mu \nu} \frac{d x_{\nu}}{d \tau}+\frac{2}{3} \frac{e^{2}}{c^{3}}\left(\frac{d^{3} x^{\mu}}{d \tau^{3}}+\frac{1}{c^{2}} \frac{d x^{\mu}}{d \tau} \frac{d^{2} x^{\nu}}{d \tau^{2}} \frac{d^{2} x_{\nu}}{d \tau^{2}}\right)+e F_{Z P}^{\mu \nu} \frac{d x_{\nu}}{d \tau}
$$

where $F_{C o u l}^{\mu \nu}$ gives the electromagnetic fields of the Coulomb potential, the term involving $2 e^{2} /\left(3 c^{3}\right)$ gives the radiation damping, and $F_{z p}^{\mu \nu}$ is the random zero-point radiation with the correlation function given in Eq. (14). The scattered electromagnetic fields can be written in terms of the potentials as $F^{\mu \nu}=\partial^{\mu} A^{\nu}-\partial^{\nu} A^{\mu}$, where the potentials satisfy the wave equation with source $J^{\mu}$ given by the scattering charged particle

$$
\square^{2} A^{\mu}=\frac{4 \pi}{c} J^{\mu}
$$

with solution

$$
A^{\mu}(x)=A^{i n \mu}(x)+\frac{1}{c^{2}} \int d^{4} x^{\prime} D\left(x-x^{\prime}\right) J^{\mu}
$$

where $A^{i n \mu}$ is the vector potential for the incoming zero-point radiation $F_{z p}^{\mu \nu}=\partial^{\mu} A^{i n \nu}-$ $\partial^{\nu} A^{i n \mu}$, while $D\left(x-x^{\prime}\right)=2 c \theta\left(x^{0}-x^{\prime 0}\right) \delta\left[\left(x-x^{\prime}\right)^{2}\right]$ is the retarded Green function for the scalar wave equation, and $J^{\mu}$ is the current density for the scattering charged particle $J^{\mu}(x)=e c \int d \tau\left(d x_{e}^{\mu} / d \tau\right) \delta^{4}\left[x-x_{e}(\tau)\right]$.

Since under a scaling transformation, the mass $m$ transforms inversely with $\sigma_{l t E^{-1}}$, while $x^{\mu}$ and $\tau$ transform directly with $\sigma_{l t E^{-1}}$, it follows that every term in Eq. (15) scales as $\sigma_{l t E^{-1}}^{-2}$ 



wave equation (16) involves three inverse powers of $\sigma_{l t E^{-1}}$ on each side and hence is $\sigma_{l t E^{-1}}$ scale invariant. Similarly, every term in Eq. (17) scales as $\sigma_{l t E^{-1}}^{-1}$ and hence that equation is also $\sigma_{l t E^{-1}}$-scale invariant. Since the scattering equations are scale invariant provided the

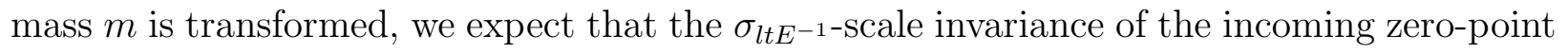
radiation will be preserved for the Coulomb potential, just as the spectrum in our model

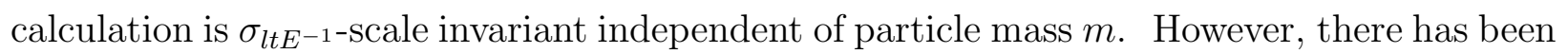
no complete calculation showing the invariance of zero-point radiation under scattering by a charged particle in a Coulomb potential.

In the physics literature, all of the scattering calculations for random classical radiation involve potentials other than the Coulomb potential. In the inertial frame where the radial force on the orbiting particle takes the form $F=n k / r^{n+1}$, the particle equation of motion corresponding to Eq. (15) for a general potential function will not be $\sigma_{l t E^{-1} \text {-scale invariant }}$ since invariance in form requires that the constant $k$ be transformed with $1-n$ factors of $\sigma_{l t E^{-1}}$, as in Eq. (9). Since for a general potential function the equations describing the

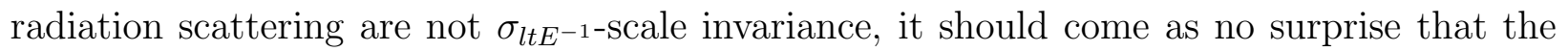

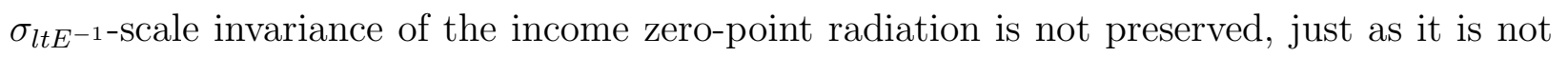
preserved in our model calculation if $n \neq 1$. Rather, the Rayleigh-Jeans spectrum, which is not $\sigma_{l t E^{-1} \text {-scale invariant, seems to be the spectrum which is invariant for (at least dipole) }}$ scattering by more general classical potentials. [2] [3] [4]

It is also worth noting that zero-point radiation is the unique spectrum of random radiation which is Lorentz invariant.[11] Equation (14) gives the manifestly Lorentz covariant two-point correlation function for classical electromagnetic zero-point radiation. In Eqs. (15)-(17) we have given the manifestly Lorentz-covariant form for the scattering equations. We suggest again that the Lorentz-invariant character of zero-point radiation is likely to be preserved by such a scattering system. On the other hand, a general mechanical scatterer breaks the Lorentz-invariance of the scattering equations. It does not seem surprising that the Lorentz-invariant zero-point spectrum is transformed toward a non-Lorentz-invariant form by scattering systems which are not Lorentz invariant. 


\section{CONCLUSION}

In this article we have carried out a specific model calculation for the coherent electromagnetic radiation spectrum of minimum intensity which will hold a charged particle in a circular orbit in a potential function $V(r)=-k / r^{n}$. We find that the radiation spectrum

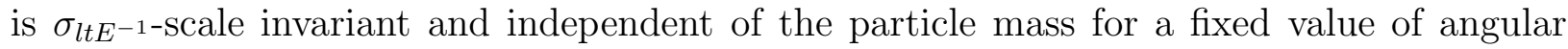
momentum only in the case where the Coulomb potential with fixed charge $e^{2}$ provides the

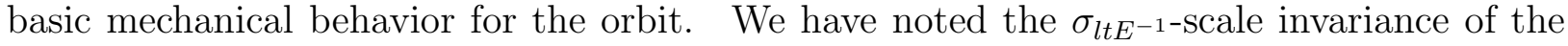
electromagnetic radiation and the scattering equations for electromagnetic radiation when the Coulomb potential is used. We believe that our arguments strongly suggest that the Coulomb potential may provide an electromagnetic scatterer which leaves invariant the zeropoint radiation spectrum. Rather than being merely a "loophole" in the problem of classical radiation equilibrium, this calculation is the fundamental classical calculation which needs to be performed.

However, nonrelativistic quantum physics apparently has such a complete hold on the minds of many physicists, that they reject the possibility that some classical calculations still need to be done to clarify the distinctions between classical and quantum phenomena.

[1] T. H. Boyer, "Random electrodynamics: The theory of classical electrodynamics with classical electromagnetic zero-point radiation," Phys. Rev. D 11, 790-808 (1975). See the appendix for the stability of the zero-point radiation under scattering by an electric dipole oscillator.

[2] J. H. Van Vleck, "The Absorption of Radiation by Multiply Periodic Orbits, and its Relation to the Correspondence Principle and the Rayleigh-Jeans Law. Part II Calculation of Absorption by Multiply Periodic Orbits," Phys. Rev. 24, 347-365 (1924).

[3] T. H. Boyer, "Equilibrium of random classical electromagnetic radiation in the presence of a nonrelativistic nonlinear electric dipole oscillator," Phys. Rev. D 13, 2832-2845 (1976). T. H. Boyer, "Statistical equilibrium of nonrelativistic multiply periodic classical systems and random classical electromagnetic radiation," Phys. Rev. A 18, 1228-1237 (1978).

[4] R. Blanco, L. Pesquera, and E. Santos, "Equilibrium between radiation and matter for classical relativistic multiperiodic systems. Derivation of Maxwell-Boltzmann distribution from 
Rayleigh-Jeans spectrum," Phys. Rev. D 27, 1254-1287 (1983); "Equilibrium between radiation and matter for classical relativistic multiperiodic systems II. Study of radiative equilibrium with Rayleigh-Jeans radiation," Phys. Rev. D 29, 2240-2254 (1984). These articles suggest that a relativistic scatterer again leads to the Rayleigh-Jeans spectrum as the equilibrium spectrum for classical radiation. The calculations involve the use of the relativistic expression for the linear momentum of the orbiting particle in a general class of potentials $V(r)$ which excludes the Coulomb potential. Such systems are not relativistic systems. Only the Coulomb potential has been extended to fully relativistic theory. This question of obtaining relativistic theories from mechanical potentials is discussed by T. H. Boyer, "Concerning Potential Functions in Relativistic and Nonrelativistic Accelerated Coordinate Frames," submitted for publication.

[5] T. H. Boyer, "Connecting blackbody radiation, relativity, and discrete charge in classical electrodynamics," Found. Phys. 37, 999-1026 (2007). (physics/0605003)

[6] M. J. Sparnaay, "Measurement of the attractive forces between flat plates," Physica 24, 751764 (1958). S. K. Lamoreaux, "Demonstration of the Casimir force in the 0.6 to $6 \mu \mathrm{m}$ range," Phys. Rev. Lett. 78, 5-8 (1997); 81, 5475-5476 (1998). U. Mohideen, "Precision measurement of the Casimir force from 0.1 to $0.9 \mu \mathrm{m}$," Phys. Rev. Lett. 81, 4549-4552 (1998). H. B. Chan, V. A. Aksyuk, R. H. Keiman, and F. Capasso, "Quantum mechanical actuation of microelectromechanical systems by the Casimir force," Science 291, 1941-1944 (2001). G. Bressi, G. Carugno, R. Onofrio, and G. Ruoso, "Measurement of the Casimir force between parallel metallic surfaces," Phys. Rev. Lett. 88, 0441804(4) (2002).

[7] T. H. Boyer, "Diamagnetism of a free particle in classical electron theory with classical electromagnetic zero-point radiation," Phys. Rev. A 21, 66-72 (1980).

[8] T. H. Boyer, "Thermal effects of acceleration through random classical radiation," Phys. Rev. D 21, 2137-2148 (1980); "Thermal effects of acceleration for a classical dipole oscillator in classical electromagnetic zero-point radiation," Phys. Rev. D 29, 1089-1095 (1984).

[9] See for example, T. H. Boyer, "Van der Waals Forces and Zero-Point Energy for Dielectric and Permeable Materials," Phys. Rev. A 9, 2078-2084 (1974) and "Temperature Dependence of Van Der Waals Forces in Classical Electrodynamics," Phys. Rev. A 11, 1650-1663 (1975)

[10] The classical constant $b_{z p}$ clearly takes the same value as $(1 / 2) \hbar$. However, $b_{z p}$ enters in purely classical electromagnetic theory and has nothing to do with ideas of energy quanta. 
[11] T. W. Marshall, "Statistical Electrodynamics," Proc. Camb. Phil. Soc. 61, 537-546 (1965); T. H. Boyer, "Derivation of the Blackbody Radiation Spectrum without Quantum Assumptions," Phys. Rev. 182, 1374-1383 (1969).

[12] T. H. Boyer, "Scaling Symmetry and Thermodynamic Equilibrium for Classical Electromagnetic Radiation," Found. Phys. 19, 1371-1383 (1989).

[13] T. H. Boyer, "Conformal Symmetry of Classical Electromagnetic Zero-Point Radiation," Found. Phys. 19, 349-365 (1989).

[14] See, for example, E. A. Power, Introductory Quantum Electrodynamics (American Elsevier, New York 1964), pp. 18-22.

[15] J. D. Jackson, Classical Electrodynamics, 2nd. ed., (Wiley, New York 1975), p. 665.

[16] See for example, C. Teitelboim, D. Villarroel, and Ch. G. van Weert, "Classical Electrodynamics of Retarded Fields and Point Particles," Riv. de Nuovo Cimento 3, 1-64 (1980). 